\title{
Language Proficiency and Media Synchronicity Theory: The Impact of Media Capabilities on Satisfaction and Inclusion in Multilingual Virtual Teams
}

\author{
A. Carolin Fleischmann \\ University of Southern California \\ fleischc@marshall.usc.edu
}

\author{
Jolanta Aritz \\ University of Southern California \\ aritz@marshall.usc.edu
}

\author{
Peter Cardon \\ University of Southern California \\ cardon@marshall.usc.edu
}

\begin{abstract}
Virtual teams that use integrated communication technologies are ubiquitous in cross-border collaboration. This study explored media use and communication performance in multilingual virtual teams. Based on surveys from 96 virtual teams (with 578 team members), the research showed that more time spent in synchronous communication channels such as online conferences increased inclusion and satisfaction. Team members with lower language proficiency felt less included in synchronous and asynchronous collaboration, whereas team members with higher language proficiency felt less satisfied with asynchronous collaboration. Also, limited language proficiency speakers were significantly less likely to view synchronous tools as helpful for their teams to reach a mutual decision. Our data supports Media Synchronicity Theory (MST) for native and highly proficient English speakers. However, MST needs to be adjusted to account for different levels of language proficiency.
\end{abstract}

\section{Introduction}

Companies are increasingly relying on virtual teams to bring together the necessary expertise needed to tackle complex global problems and arrive at highquality decisions. Virtual teams are characterized by their reliance on collaboration technologies to achieve their mutual goal. Additionally, virtual team members are geographically dispersed; oftentimes across borders and time zones [1]. Multilingual virtual teams in particular face communication challenges that may impact their inclusion and satisfaction and ultimately team performance. The quality of multilingual team communication processes depends on the media that is being used for a specific task.

Cross-border virtual teamwork has been widely studied over the past decade. Furthermore, media synchronicity theory (MST) has been widely used to explore technology-mediated communication. MST "proposes that communication performance will improve when the needs of conveyance and convergence processes are matched to appropriate media” (p. 592) [2].Yet, research about appropriate media use and communication performance based on media use by virtual team members of varying degrees of language proficiency is relatively limited. Furthermore, the degree to which language proficiency supports or counters MST propositions is not entirely understood [3], [4]. Finally, it's not clear how the intersections of language proficiency and media use impact team inclusion and team satisfaction.

With a robust sample of global virtual teams, our research aims to explore whether the assumptions of MST hold for multilingual settings. We hypothesize that this is the case for conveyance of information. For convergence of information, however, team members with lower level of language proficiency are expected to perform better on asynchronous media - contrary to MST's assumptions.

Furthermore, we assess the level of inclusion and satisfaction of team members when using different communication media. We expect both satisfaction and inclusion to be higher when using richer, synchronous communication channels. We anticipate that this effect is be stronger for team members with a high level of language proficiency.

Our study contributes to the body of research on communication media in virtual teams by adding the dimension of language proficiency to MST. Specifically, our contribution is threefold: First, our study advances research by showing which communication channels team members of differing levels of language proficiency consider effective for conveyance and convergence of meaning in multilingual virtual teams. Second, we include inclusion and satisfaction as variables of communication performance and show that synchronous communication increases feelings of inclusion and satisfaction within a multilingual virtual team. Lastly, our study provides evidence that team 
members of different levels of language proficiency do not feel equally included and satisfied in synchronous and asynchronous communication.

\section{Theoretical Background}

Three areas of research are relevant for developing our hypotheses: Media synchronicity theory and virtual teams (2.1), language in virtual teams (2.2), and satisfaction and inclusion in virtual teamwork (2.3).

\subsection{Media synchronicity theory (MST) and virtual teams}

MST distinguishes between synchronous channels, such as video conferencing, and asynchronous channels, such as e-mail.

The theory proposes that the communication effectiveness is enhanced when the synchronicity of a given medium matches the synchronicity that a communication process requires. MST contends that communication consists of two different processes: conveyance and convergence of meaning. Conveyance of meaning is information transmission from one team member to the other. Convergence of meaning, on the other hand, requires negotiating a common understanding of information and requires going back and forth between team members. The communication needs of the task influence the appropriation and use of media, which in turn influence communication performance. According to MST, conveyance processes rely on lean media with lower synchronicity whereas convergence requires medium with higher synchronicity to achieve more effective communication. In most communication technologies, highly synchronous media are characterized by immediacy of transmission, social presence, and a variety of symbol sets, including auditory and visual cues. Asynchronous media allow for parallelism of information transmission, rehearsability, and reprocessability. Many studies have looked at the various factors that affect the communication needs and determine the performance of different channels to accomplish the task [2].

Bartelt and Dennis found that team performance and decision-making were influenced not just by which tool the team used, but the genre rules or the social structures for using a communication technology [5]. Windeler and Harrison investigated cooperation and found that MST explained communication and task performance in a cooperative context but was insufficient to capture how media capabilities influence performance in a noncooperative context [6].

Research on swift teams found that contrary to the expectation to rely on highly synchronous media for maximum coordination, swift teams shifted from highly synchronous media in the beginning stages of the performance to asynchronous media in the later teaming stages, and the effect is intensified in high performing teams [7]. Fuller et al. found that CMC anxious individuals participated less and were ranked more poorly by their team members in virtual team environment. Interestingly, CMC-anxious participants did not improve with repeated CMC experiences [8].

Aritz et al. researched the use of various media in virtual teams and found that team coordination varied depending on what media channels the team used. Well-coordinated teams used richer communication channels early on in the project. Members of less coordinated and poorly coordinated teams started with the assumption that traditional, less rich tools such as email would be more effective than social networking tools. At the end of the project, team members identified rich and social channels as more effective [9].

Yet, while these handful of studies exist about MST and virtual teams, few studies explore MST in virtual teams focusing on contemporary communication platforms. Rather, the majority of studies continue to focus on traditional options, such as e-mail and chat (e.g., [10], [11]), while multiple new CMCs exist (e.g., meeting tools, social networking), few have received research attention in the context of MST [12]. Not surprisingly, scholars such as DeLuca and Valacich call for more studies to see if MST holds across different environments and how they might affect the choice of medium to accomplish different tasks [13]. Other than new collaboration platforms, neglected environments are cross-border, and more specifically, multilingual teams.

\subsection{Language in virtual teams}

Language has traditionally been researched by linguists. In recent years, business communication and international business have seen a fast growing number of publications in the field of language use in business [14]. However, studies on the implications of language proficiency in technology-mediated communication are scarce. At the same time, business communication relies heavily on technology. This is particularly true for cross-border business communication which is oftentimes characterized by linguistic differences and geographical dispersion.

Many studies focus on English as the language of choice in business environments and research 
corporate language policies [15] or Business English as a lingua franca (BELF) [16]. BELF is sometimes considered to be a hybridized language that is neutral in the sense that it is not owned or influenced by a specific culture [17], [18]. It may even include elements from other languages and emerge from the co-existence of multiple languages in multinational corporations (MNC), thus mingling and forming 'linguascapes' [19].

At the team level, most studies research collocated teams (e.g. [20]). These span from teams of construction workers and safety issues due to language-related miscommunication [21] to top management teams and their quality of discussions [22]. Studies focus on the impact of language diversity on trust, cohesion, and conflict (e.g. [23], [18]) and relationships of power and status (e.g. [24]).

In the context of cross-border virtual team collaboration, Hinds, Neeley, and Cramton identify language-related fault lines that may lead to power struggles and subgroup formation in global teams [25]. Cohen and Kassis-Henderson outline the positive effects of language diversity by pointing out that team members - while communicating in English as a lingua franca - draw on their multilingual backgrounds to encode and decode meaning [26].

Some researchers suggest that new media create challenges for less proficient English speakers. For example, Orta-Castañon et al. reported that communication became more difficult even with the social collaboration platforms, such as Facebook, Skype, Whatsapp, etc. when virtual teams included more languages and time zones and differing degrees of English language proficiency [27].

The specific perspective on the technology that multilingual virtual teams use for communicating is still scarce. The existing, exclusively qualitative studies address media choice's influence on multilingual team processes and outcomes. Klitmøller, Schneider, and Jonsen find that social categorization is an issue for multilingual virtual teams only when they are using oral communication channels. They did not find effects of social categorization when teams were using written communication [4]. Klitmøller and Lauring distinguished different technology-mediated communication channels by drawing on MRT. They argue that in linguistically diverse teams, lean media should be used for effectively sharing complex knowledge that is prone to differing interpretations according to context. In contrast, they recommend rich media for sharing canonical knowledge which does not leave much room for interpretation [3]. These findings question some of the assumptions of MRT and MST, leading to the conclusion that adjustments to these theories are necessary in a multilingual context [28]. This is particularly true for the convergence of meaning. Multilingual teams do not benefit from the back-and-forth of synchronous media to negotiate a shared understanding. Team members with limited language proficiency struggle with instant communication in a foreign language, thus demanding an increase in cognitive effort and allocating a substantial amount of their cognitive capacity to language processing instead of task processing. They therefore prefer asynchronous over synchronous channels as an easier way of communicating that allows them to focus their cognitive efforts on the task at hand [28].

Based on the qualitative research about language proficiency and MST, we propose the following hypotheses:

H1: For conveyance, virtual team members with lower language proficiency will more likely consider asynchronous communication channels effective.

H2: For convergence, virtual team members with lower language proficiency will more likely consider asynchronous communication channels effective.

\subsection{Inclusion and satisfaction in virtual teamwork}

Communication media and language diversity are both factors that have not been thoroughly researched in the context of team member well-being [12]. Some research, however, suggests that communication media perform differently in satisfying and including team members varying levels of language proficiency.

The impact of media on communication performance is contingent on members' proficiency in the team's working language. Multilingual team members use differing sets of symbols to encode and decode messages. Language diversity may increase misunderstandings, loss of information, and noncommunication. Similar issues have been diagnosed in technology-mediated team collaboration in general [29].

According to team effectiveness models (e.g. [30]), team outcomes have two components: In addition to team performance, which is the level of task accomplishment, social outcomes include team member satisfaction with the overall task, process, and result, and team members' sense of inclusion, value, and support in the process. Satisfaction and team performance are correlated and have been found to influence each other [31]. This paper focusses on the 
social outcomes of language proficiency and how different media foster communication performance: the sense of inclusion and satisfaction.

The use of rich and synchronous media has been associated with satisfied team members. Media that allows the transmission of rich, visual cues promotes a shared understanding which in turn influences satisfaction positively. This relationship has been shown for collaborative visualization techniques [32]. Team inclusion, as another important social outcome, is particularly fostered when using community-based communication media, such as discussion forums or videoconferencing, instead of personalized communication media, such as email [33]. These richer, more synchronous, and community-based media are particularly important in virtual team inclusion [9].

H3: More frequent use of synchronous communication channels (i.e., online conferences, messaging) will lead to a higher sense of inclusion and satisfaction.

When studying multilingual teams, team members with limited language proficiency are more likely to feel excluded. The excluded individual is impacted in his or her ability to contribute ideas and thoughts to the group discussion; therefore, decreasing potential team performance. Inclusive communication strategies, such as the use of redundancies or probing for understanding, are even more important in linguistically diverse contexts [34]. These strategies ensure effective communication in a sense that all team members are included and a shared understanding of tasks, objectives, and processes is created.

Feelings of exclusion are common in multilingual virtual teams, ultimately leading to negative perceptions about the teamwork and dissatisfaction. For team members with low language proficiency levels, both understanding and expression of meaning is more difficult and less effective in synchronous communication than in asynchronous communication due to time and cognitive effort needed for processing information. Hence, team members with lower proficiency levels are expected to be less satisfied synchronous communication.

We propose the following hypotheses at the intersection of language proficiency, satisfaction and inclusion, and MST:

H4a: Lower language proficiency leads to a lesser sense of inclusion in asynchronous and synchronous collaboration.
$H 4 b$ : Lower language proficiency leads to a lesser sense of satisfaction in asynchronous and synchronous collaboration.

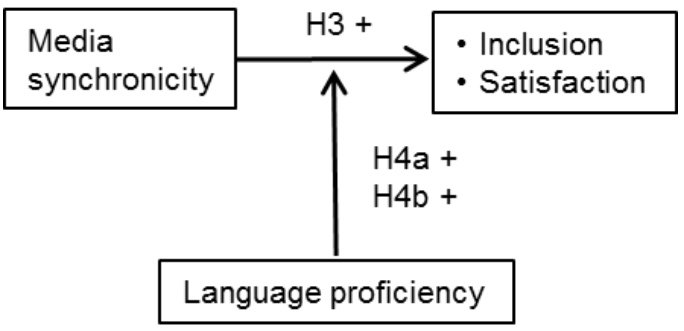

Figure 1. Research model for measuring the impact of media synchronicity and language proficiency on inclusion and satisfaction

\section{Methodology}

\subsection{Data}

We ran a virtual team project simulation that mirrors current organizational realities that involve the use of technology to collaborate with co-workers dispersed around the globe. Business students worked in multilingual virtual teams. The teams were instructed to use a single collaboration platform provided for the project.

Virtual team simulation participants were placed in teams from different institutions and never meet in person. They used the IBM Connections platform (an enterprise social networking platform with tools such as online conferences, email, messaging, forums, and shared files). This study involved participants enrolled in business communication courses during the Spring Semester 2018 from 14 universities in seven countries, including the United States, India, Canada, Germany, France, Spain, and Finland. A total of 96 teams of 578 graduate and undergraduate students participated in the study. The project required participants to work together virtually to research and analyze an organization's social media presence and then to collaboratively write a report on their findings. Students worked in diverse teams of 5 or 6 members. The teams were combined to ensure roughly equal diversity. On average, each team had three U.S.-based students - from different institutions - and three nonU.S.-based students from different countries. Altogether, the project spanned six weeks.

Participants were asked to complete a survey at the end of the project. The survey was available in English language, because English was the teams' working language and a single-version questionnaire guarantees sematic equivalence. However, differences 
in language proficiency and language background may influence understanding and interpretation of concepts in the survey. Of the 578 participants, 451 completed the surveys for a response rate of $78 \%$.

\subsection{Measures}

To explore our hypotheses, we used measures for (a) language proficiency, (b) use of communication channels to achieve conveyance and convergence of meaning, (c) frequency of use for various communication channels, and (d) overall team inclusion and team satisfaction.

Many measures of language proficiency simply distinguish between native speakers and non-native speakers. This simplistic distinction neglects the complexity of language. Language is not a fixed entity, but rather has an inherent diversity within. This diversity becomes apparent in a variety of dialects, accents, and lexical and grammatical usages [26].

Our study uses perceived measures of language proficiency, because English used in professional settings differs from a standardized national code. Workplace communication performs a social function and builds relationships between colleagues, corporate culture, and work climate [35], [36]. Formal language performance measures often do not reflect the conversational ability and cultural and pragmatic norms to use the language and therefore are often not accurate measures of a person's working ability to use the language [37]. Perceived language proficiency is crucial for the mutual understanding between team members and their social relationship [18].

As a result, we used team member ratings of one another (on a 7-point scale) to measure language proficiency and computed language proficiency as the mean of all ratings. In the ANOVA analysis used to address the first two hypotheses, we distinguish between native (perfect/near-perfect peer ratings), high proficiency (average ratings above 6), and limited proficiency (average ratings below 6). For the regression analysis to address hypotheses 3 and 4, we used a continuous measure of language proficiency.

To measure the effectiveness of various communication channels to reach conveyance and convergence, participants responded to the following questions: Please rank in order of importance $(1=$ most important) what type of communication you preferred to use to exchange information (conveyance): file sharing, forums, email, texting or messaging, social networking, and online conferences; Please rank in order of importance (1=most important) what type of communication you preferred to use to come to consensus and make decisions (convergence): file sharing, forums, email, texting or messaging, social networking, and online conferences.

Additionally, participants responded to the following question about the frequency with which their teams various communication channels: Which media did you use to communicate with your teammates? For each communication channel (file sharing, forums, email, texting or messaging, social networking, and online conferences), participants responded on the following scale: $1=$ never to $7=$ all the time. The collaboration platform enables accessing all past team communication which helped minimize recall bias.

Finally, we used two sets of questions to measure students' sense of 1) inclusion in teamwork and 2) satisfaction with team performance based on the work of Aritz et al. [9]. Three questions addressed students' sense of inclusion in team decision-making, using a 5-point Likert scale: a) I was included in the group discussion and decision making; b) I was valued for my contributions to the group discussion and decision making; c) My group members supported me and my ideas. Three questions measured satisfaction with team performance, using a 5-point Likert scale: a) I am satisfied with the final result we produced; b) I am satisfied with my team's performance; c) I am satisfied with the decisions my team made [38]. We modified these items to address both synchronous and asynchronous collaboration. These inclusion and satisfaction items were preceded by the following: (a) Now think about your team communication when you were holding real-time online (video) meetings and (b) Now think about your team's written communication. Thus, these items resulted in team measures of (1) inclusion in synchronous collaboration; (2) inclusion in asynchronous collaboration; (3) satisfaction with synchronous collaboration; and (4) satisfaction with asynchronous collaboration.

\section{Results}

We first tested $\mathrm{H} 1$ and $\mathrm{H} 2$ which focused on the perceived performance of different communication channels for conveyance and convergence of meaning. We found little difference among various levels of language proficiency based on analysis of variance (ANOVA). All except one ANOVA are nonsignificant $(\mathrm{p} \geq 0.1)$.

Our findings show that virtual team members of varying levels of language proficiency will equally rank the effectiveness of communication channels for conveyance. No clear preference for asynchronous communication in conveyance of information - as proposed by MST - emerges, neither for high nor for 
low language proficiency team members. Therefore, $\mathrm{H} 1$ is not supported by our data.

The one major exception to these equal ratings of communication performance was the use of online conferences ( $p=0.005$ ) for convergence of meaning. With average rankings of 2.3 and 2.1, native and high proficiency
English speakers ranked online conferences as critical for convergence; limited proficiency English speakers were significantly less likely to rank online conferences as highly $(M=2.7)$. Yet, there were no significant differences among the various asynchronous channels. Thus, we consider H2 partially supported.

\section{Table 1. Perceived communication performance for conveyance and convergence at different}

levels of language proficiency

\begin{tabular}{|c|c|c|c|c|c|}
\hline & \multicolumn{3}{|c|}{ English Language Proficiency } & \multirow[b]{3}{*}{$F$} & \multirow[b]{3}{*}{$p$} \\
\hline & Native & High & Limited & & \\
\hline & $M(S D)$ & $M(S D)$ & $M(S D)$ & & \\
\hline \multicolumn{6}{|l|}{ Conveyance } \\
\hline File Sharing & $2.8(1.4)$ & $2.8(1.3)$ & $3.0(1.4)$ & 1.1 & .33 \\
\hline Forums & $3.4(1.6)$ & $3.4(1.6)$ & $3.6(1.6)$ & 0.3 & .75 \\
\hline Email & $3.9(1.4)$ & $4.2(1.4)$ & $4.0(1.4)$ & 1.3 & .28 \\
\hline Messaging* & $3.1(1.8)$ & $3.1(1.8)$ & $3.2(1.8)$ & 0.2 & .83 \\
\hline Social Networking & $4.5(1.7)$ & $4.5(1.7)$ & $4.2(1.8)$ & 1.5 & .23 \\
\hline Online Conferences* & $3.0(1.4)$ & $2.8(1.3)$ & $2.8(1.4)$ & 0.8 & .46 \\
\hline \multicolumn{6}{|l|}{ Convergence } \\
\hline File Sharing & $3.4(1.3)$ & $3.3(1.2)$ & $3.5(1.3)$ & 0.5 & .61 \\
\hline Forums & $3.4(1.4)$ & $3.2(1.5)$ & $3.3(1.6)$ & 0.3 & .74 \\
\hline Email & $4.0(1.3)$ & $4.2(1.3)$ & $4.0(1.4)$ & 1.1 & .34 \\
\hline Messaging* & $3.1(1.7)$ & $3.1(1.7)$ & $3.0(1.6)$ & 0.1 & .92 \\
\hline Social Networking & $4.6(1.7)$ & $4.7(1.5)$ & $4.3(1.8)$ & 2.3 & .10 \\
\hline Online Conferences* & $2.3(1.6)$ & $2.1(1.4)$ & $2.7(1.7)$ & 5.3 & .005 \\
\hline
\end{tabular}

${ }^{*}$ Study participants perceive media with an asterisk as synchronous.

To test hypotheses 3 and 4, we conducted multivariate OLS regression analyses (see Table 2). Social networking and file sharing were excluded, because the included channels are all part of a social collaboration tool. In addition, file sharing was considered a very functional and outcome oriented medium and therefore less relevant for inclusion and satisfaction.

More time spent in online conferences overwhelmingly increased inclusion and satisfaction, strongly supporting our third hypothesis. Online conferences were highly significant predictors in all four models in Table 2 (e.g. $\beta=0.237, p<0.001$ for Inclusion in Asynchronous Communication). The increased use of messaging - another synchronous communication channel - likewise contributed to higher inclusion in synchronous communication $(\beta=$ 0.106, $p<0.05$ ) and higher satisfaction for all communication $(\beta=0.175, p<0.005$ and $\beta=0.199$, $p$ $<0.001)$.

Finally, we found that English proficiency significantly impacted virtual team members' sense of inclusion and their satisfaction with team performance. Individuals with higher English proficiency were more likely to feel included in both synchronous $(\beta=0.176, p<0.001)$ and asynchronous $(\beta=0.147, p<0.005)$ communication, confirming H4a. However, the opposite was the case for satisfaction, disconfirming and even showing the opposite of H4b. Individuals with less proficiency were more likely to be satisfied with the team's performance in asynchronous communication $(\beta=$ 0.108, $p<0.05$ ).

In summary, our data supports H3 and H4a. It partially supports H2. H1 and H5b are not supported. For H5b, data shows the opposite relationship. 
Table 2. Impacts of English proficiency and media on inclusion and satisfaction

\begin{tabular}{lcccc}
\hline & \multicolumn{2}{c}{ Inclusion in } & \multicolumn{2}{c}{ Satisfaction with } \\
\cline { 2 - 5 } & $\begin{array}{c}\text { Asynchronous } \\
\text { Communication }\end{array}$ & $\begin{array}{c}\text { Synchronous } \\
\text { Communication }\end{array}$ & $\begin{array}{c}\text { Asynchronous } \\
\text { Communication }\end{array}$ & $\begin{array}{c}\text { Synchronous } \\
\text { Communication }\end{array}$ \\
\hline English proficiency & $.147^{* *}$ & $.176^{* * *}$ & $-.108^{*}$ & -.052 \\
Online conferences & $.237^{* * *}$ & $.240^{* * *}$ & $.161^{* *}$ & $.202^{* * *}$ \\
Messaging & .081 & $.106^{*}$ & $.175^{* *}$ & $.199^{* * *}$ \\
Forums & .073 & .037 & .049 & .076 \\
Email & $-.104^{*}$ & .022 & -.042 & .011 \\
\hline estimated $\beta$ values are reported & & & \\
${ }^{* * *} \mathrm{p}<0.001,{ }^{* *} \mathrm{p}<0.005,{ }^{*} \mathrm{p}<0.05$ & & &
\end{tabular}

\section{Discussion}

This study showed that team members with limited language proficiency consider different media effective compared with those of higher and native proficiency. Our study contributes to the advancement of theory by suggesting that MST should be modified to address language proficiency and modern collaboration tools. Furthermore, our study advances research of social outcomes of virtual team collaboration by showing that media use in multilingual virtual teams can significantly impact team inclusion and satisfaction.

MST proposes that synchronous media are more suitable for convergence of meaning whereas asynchronous media are more suitable for conveyance purposes. Siemon et al. confirmed this proposition: Synchronous, collaborative visualization increases shared understanding in a team [32]. Additionally, team members who are not familiar with each other nor with the media, have a higher need for synchronous media regardless of the task at hand [2]. The teams in our sample work together for a short time, have diverse backgrounds, and use the IBM Connections collaboration platform for the first time. The positive view on synchronous communication for both conveyance and convergence of meaning is therefore lending support for MST and in line with previous research.

Yet, our study also revealed some potential adjustments that have implications for the advancement of MST theory, specifically related to language proficiency in virtual teams. Speakers with limited language proficiency are less likely to consider synchronous communication channels effective for convergence of meaning. In line with the research of Klitmøller and Lauring [3] but contrary to the proposition of MST, limited proficiency speakers rank synchronous communication highest for conveyance of information. Highly proficient team members rank synchronous communication as most effective for convergence. Thus, our data supports MST for native and high proficiency team members. However, adjustments to the theory are needed for lower proficiency team members: MST should explicitly include language proficiency as an appropriation factor that influences the use of communication technology. For low proficiency team members, synchronous communication is not as effective for convergence of meaning as for native and high proficiency team members

One of our strongest conclusions is that more frequent use of synchronous channels is critical to team inclusion and satisfaction. Synchronous communication increases shared understanding which in turn increases satisfaction [32]. Contrary to the findings of Colazo that asynchronous tools were used and prioritized in higher-performing virtual teams [7], our study showed that more frequent use of synchronous tools significantly and positively impacted team inclusion and satisfaction in both synchronous and asynchronous collaboration. Specifically, online conferences were positively connected in all forms of inclusion and satisfaction. Messaging was also connected to inclusion for synchronous collaboration and satisfaction with synchronous and asynchronous collaboration. While there's some debate about whether messaging is synchronous or asynchronous [9], this research seems to place messaging closer to synchronous communication. On the other hand, email, as an asynchronous and personalized rather than community-based communication channel, was detrimental to team inclusion.

Furthermore, virtual team members of lower language proficiency tend to feel less included in synchronous and asynchronous collaboration. While higher levels of inclusion for high proficiency 
speakers were expected, we found an opposite relationship between language proficiency and satisfaction which is somewhat perplexing. Contrary to Tenzer and Pudelko's finding that team members with lower language proficiency prefer asynchronous over synchronous communication [28], language proficiency did not have an influence on satisfaction with synchronous communication in our sample.

However, virtual team members of lower language proficiency were more likely to be satisfied with asynchronous collaboration than their higher proficiency teammates. Asynchronous communication is mostly written communication. With the deliverable of the team project - the output of synchronous and asynchronous team communication - being a written report, team members with lower proficiency benefitted from the written communication skills of their native teammates which in turn increased their level of satisfaction with written (asynchronous) communication. Additionally, lower language proficiency team members may have felt more at ease in asynchronous communication, because that allowed rehearsing and reprocessing the message. They are therefore more satisfied with asynchronous communication than native and highly proficient speakers who are less satisfied with asynchronous communication because it takes more time due to lower transmission velocity and misunderstandings cannot be clarified immediately due to a limited symbol set (e.g. lack of hand gestures and facial expressions).

\section{Limitations and directions for further research}

As some of our findings are contradictory to MST and evidence in the literature, more research is needed to understand the causes behind the relationships between language proficiency, MST, and inclusion and satisfaction. Future research needs to examine additional factors, such as team members' capabilities relevant to the task and outcome of the task to further understand the role of language proficiency that extends beyond social outcomes.

Team members in our sample perceive messaging as a form of synchronous communication. Yet, it is written communication and therefore possesses some of the characteristics of asynchronous communication. The same is true for forums and file sharing when synchronous editing is enabled. Further research needs to specifically address integrated collaboration platforms as a way to overcome language barriers in cross-border virtual collaboration.
Our research has limitations concerning sample characteristics. We aim at making recommendations for multilingual virtual teams in a business context. Therefore, a survey in a corporate context would be beneficial. This would likely allow a more differentiated view on multilingual virtual teams' communication challenges, because - among other factors - the average age of participants would increase. Age may have implications on the familiarity with new media and the level of language proficiency thus making synchronous communication even more challenging and the results of this research even more conclusive.

Our research also does not account for how participants have used media in prior projects. Research suggests that these habits may influence communication performance in virtual teams. We recommend future research that accounts for these media habits, particularly for cross-cultural virtual teams. Future research should also control for colocated teams working on the same platform as well as co-located teams working non-virtually.

Finally, we recommend future research about crosscultural virtual teams that is designed to account for culturally constructed views of inclusion and satisfaction.

\section{Conclusion}

This paper examined whether the assumptions of MST hold for virtual teams that are composed of team members with differing levels of language proficiency. More specifically, we assessed team members' perception of media effectiveness for conveyance and convergence of meaning. In a second step, we investigated how included and satisfied team members of differing language proficiency levels felt when using synchronous and asynchronous communication media.

Our data supports MST for native and highly proficient English speakers. However, adjustments to MST are needed for team members with limited language proficiency. They were less likely to consider synchronous communication channels effective for convergence of meaning than their highly proficient teammates. As far as communication performance, they were more satisfied with asynchronous communication and equally satisfied with synchronous communication compared to their native and high language proficiency counterparts. MST outlines rehearsability and reprocessability as advantages of asynchronous communication in virtual team collaboration. These advantages become even more relevant for team members with limited language 
proficiency. While our study indicates that highly proficient speakers still feel more included, rehearsability and reprocessability in asynchronous communication leads to high satisfaction levels of lower proficiency language speakers. However, our study also seems to imply that a recommendation towards asynchronous communication channels would neglect the needs of native and highly proficient speakers. They appear to be less satisfied with asynchronous communication and rank synchronous communication as most important for convergence of meaning.

Management should implement a collaboration infrastructure consisting of communication platforms that integrate a variety of media ranging from asynchronous to synchronous channels as a first step towards creating an integrated virtual communication environment that is suitable for different tasks and different communication needs. Management has to consider varying language proficiencies before prescribing certain media. It is necessary to cater to different communication needs and preferences to ensure growth from cross-border virtual collaboration.

\section{References}

[1] M. O'Leary and J. Cummings, "The Spatial, Temporal, and Configurational Characteristics of Geographic Dispersion in Teams," MIS Quarterly, vol. 31, no. 3, pp. 433-452, 2007.

[2] A. R. Dennis, R. M. Fuller and J. S. Valacich, "Media, tasks, and communication processes: A theory of media synchronicity," MIS Quarterly, vol. 32, no. 3, pp. 575-600., 2008.

[3] A. Klitmøller and J. Lauring, "When global virtual teams share knowledge: Media richness, cultural difference and language commonality," Journal of World Business, vol. 48, no. 3, pp. 398-406, 2013.

[4] A. Klitmøller, S. C. Schneider and K. Jonsen, "Speaking of global virtual teams: language differences, social categorization and media choice.," Personnel Review, vol. 44, no. 2, pp. 270285, 2015.

[5] V. L. Bartelt, A. R. Dennis and, "Nature and nurture: the impact of automaticity and the structuration of communication on virtual team behavior and performance," MIS Quarterly, vol. 38, no. 2, pp. 521-538, 2014.

[6] J. Windeler and A. Harrison, "Rethinking Media Synchronicity Theory: Examining the cooperative assumption," in Proceedings of the 51st Hawaii International Conference on System Sciences, Big Island, HI, 2018.

[7] J. Colazo, "The evolution of network structure and media choice in operational emergency swift teams: An exploratory study," in Proceedings of 2015 48th
Hawaii International Conference on the System Sciences (HICSS), Kauai, HI, 2015.

[8] R. M. Fuller, M. V. Chelley and S. A. Brown, "Longitudinal effects of computer-mediated communication anxiety on interaction in virtual teams," IEEE Transactions on Professional Communication, vol. 59, no. 3, pp. 166-185, 2016.

[9] J. Aritz, R. Walker and P. W. Cardon, "Media use in virtual teams of varying levels of coordination," Business and Professional Communication Quarterly, vol. 81, no. 1, pp. 222-243, 2018.

[10] C. M. Duranti and F. Carvalho de Almeida, "Is more technology better for communication in international virtual teams?," International Journal of e-Collaboration, vol. 8, no. 1, 2012.

[11] C. -P. Lin, C. -K. Chiu, S. -W. Joe and Y. -H. Tsai, "Assessing online learning ability from a social exchange perspective: A survey of virtual teams within business organizations," International Journal of Human-Computer Interaction, vol. 26, no. 9, pp. 849-867, 210.

[12] L. Gilson, M. T. Maynard, N. C. Jones-Young, M. Vartiainen and M. Hakonen, "Virtual teams research: 10 years, 10 themes, and 10 opportunities," Journal of Management, vol. 41, no. 5, pp. 1313-1337, 2015.

[13] D. DeLuca and J. S. Valacich, "Outcomes from conduct of virtual teams at two sites: Support for Media Synchronicity Theory," in Proceedings of the 38th Annual Hawaii International Conference on System Sciences, Big Island, HI, 2005.

[14] H. Tenzer, S. Terjesen and A. -W. Harzing, "Language in international business: A review and agenda for future research," Management International Review, vol. 57, no. 6, pp. 815-854, 2017.

[15] M. Latukha, A. Doleeva, M. Järlström, T. Jokenen and R. Piekkari, "Does corporate language influence career mobility? Evidence from MNCs in Russia," European Management Journal, vol. 34, no. 4, pp. 363-373, 2016.

[16] A. Kankaanranta and B. Planken, "BELF competence as business knowledge of internationally operating business professionals," International Journal of Business Communication, vol. 47, no. 4, pp. 380-407, 2010.

[17] C. Nickerson, "The death of the non-native speaker? English as a lingua franca in business communication: A research agenda," Language Teaching, vol. 48, no. 3, pp. 390-404, 2015.

[18] C. Fleischmann, L. -C. Folter and J. Aritz, "The impact of perceived foreign language proficiency on hybrid team culture," International Journal of Business Communication, 2017 (in press).

[19] M. Janssens and C. Steyaert, "Re-considering language within a cosmopolitan understanding: Toward a multilingual franca approach in 
international business studies," Journal of International Business Studies, vol. 45, no. 5, pp. 623-639, 2014.

[20] H. Tenzer, M. Pudelko and A. -W. Harzing, "The impact of language barriers on trust formation in multinational teams," Journal of International Business Studies, vol. 45, no. 5, pp. 508-535, 2014.

[21] R. Alsamadani, M. R. Hallowell, A. Javernick-Will and J. Cabello, "Relationships among language proficiency, communication patterns, and safety performance in small work crews in the United States," Journal of Construction Engineering and Management, vol. 139, no. 9, 2013.

[22] R. Piekkari, L. Oxelheim and T. Randøy, "The silent board: How language diversity may influence the work processes of corporate boards," Corporate Governance: An International Review, 2015.

[23] J. Voss, I. Albert and D. Ferring, "Language use and value orientations in multinational work teams in Luxembourg: Conflict or harmony?," International Journal of Intercultural Relations, vol. 41, pp. 192-196, 2014.

[24] C. L. Butler, "I know how! You know how! We know how! The multinational matter of language use in task teams," International Journal of Human Resources Development and Management, vol. 11, pp. 221-234, 2011.

[25] P. J. Hinds, T. B. Neeley and C. D. Cramton, "Language as a lightning rod: Power con-tests, emotion regulation, and subgroup dynamics in global teams," Journal of International Business Studies, vol. 45, no. 5, pp. 536-561, 2014.

[26] L. Cohen and J. Kassis Henderson, "Revisiting culture and language in global management teams: Toward a multilingual turn," International Journal of Cross-Cultural Management, vol. 17, no. 1, pp. 7-22, 2017.

[27] P. Orta-Castañon, P. Urbina-Coronado, H. AhuettGarza and M. Hernández-de-Menéndez, "Social collaboration software for virtual teams: Case studies," International Journal on Interactive Design and Manufacturing, vol. 12, no. 1, pp. 1524, 2018.

[28] H. Tenzer and M. Pudelko, "Media choice in multilingual virtual teams," Journal of International Business Studies, vol. 47, no. 4, pp. 427-452, 2016.

[29] H. P. Andres, "Technology-mediated collaboration, shared mental model and task performance," Journal of Organizational and End User Computing, vol. 24, pp. 64-81, 2012.

[30] J. E. McGrath, Groups: Interaction and performance, Englewood Cliffs, NJ: Prentice-Hall, 1984.

[31] L. P. Robert and S. You, "Are you satisfied yet? Shared leadership, individual trust, autonomy, and satisfaction in virtual teams," Journal of the
Association of Information Science and Technology, vol. 69, no. 4, pp. 503-513, 2018.

[32] D. Siemon, B. Redlich, C. Lattemann and S. RobraBissantz, "Forming Virtual Teams - Visualization with Digital Whiteboards to Increase Shared Understanding, Satisfaction and Perceived Effectiveness," in ICIS 2017 Proceedings, Seoul, 2017.

[33] A. Suh, K. S. Shin, M. Ahuja and M. S. Kim, "The influence of virtuality on social networks within and across work groups: A multilevel approach," Journal of Management, vol. 28, pp. 351-386, 2011.

[34] B. Du-Babock and H. Tanaka, "Leadership construction in intra-Asian English as lingua franca decision-making meetings," International Journal of Business Communication, vol. 54, no. 1, pp. 8398, 2017.

[35] P. Pullin, "Small talk, rapport, and international communicative competence," International Journal of Business Communication, vol. 47, no. 4, pp. 455476, 2010.

[36] G. F. Thomas, R. Zolin and J. L. Hartman, "The central role of communication in developing trust and its effect on employee involvement," International Journal of Business Communication, vol. 46, no. 3, pp. 287-310, 2009.

[37] C. K. Morrow, "Communicative Language Testing," in The TESOL Encyclopedia of English Language Teaching, 2018.

[38] J. Aritz and R. C. Walker, "Leadership styles in multicultural groups: Americans and East Asians working together," International Journal of Business Communication, vol. 51, no. 1, pp. 72-92, 2014. 